\title{
Kinetic Theory of Turbulence Modeling: Smallness Parameter, Scaling and Microscopic Derivation of Smagorinsky Model
}

\author{
Santosh Ansumali \\ ETH-Zürich, Department of Materials and Department of Energy Technology, \\ CH-8092 Zürich, Switzerland \\ Iliya V. Karlin \\ ETH-Zürich, Department of Materials, CH-8092 Zürich, Switzerland \\ Institute of Computational Modeling RAS, 660036 Krasnoyarsk, Russia \\ Sauro Succi \\ Istituto Applicazioni Calcolo, CNR, viale Policnico 137, 00161 Roma, Italy
}

\begin{abstract}
A mean-field approach (filtering out subgrid scales) is applied to the Boltzmann equation in order to derive a subgrid turbulence model based on kinetic theory. It is demonstrated that the only Smagorinsky type model which survives in the hydrodynamic limit on the viscosity time scale is the so-called tensor-diffusivity model. Scaling of the filter-width with Reynolds number and Knudsen number is established. This sets the first rigorous step in deriving turbulence models from kinetic theory.
\end{abstract}

\section{Introduction}

The application of a filtering procedure to equations of hydrodynamics (NavierStokes equations) in order to construct a subgrid model is often used for the turbulence modeling [1]. The aim of such models is to take into account the effects of subgrid scales as an extra stress term in the hydrodynamic equations for the resolved scale fields. Further, the subgrid scale terms should be representable in terms of the resolved fields. This procedure, like any other attempt to coarse-grain the Navier-Stokes equations, runs into the closure problem due to the nonlinearity of the equation and due to the absence of scale separation. 
On the other hand, in statistical physics, good schemes to obtain closure approximations are known for nonlinear evolution equations (with a well-defined separation of scales). Unfortunately, attempts to borrow such schemes fail for the Navier-Stokes equations. The fundamental reason for this failure of the coarse-graining procedures on the Navier-Stokes equations is the absence of scale separation. Further, the length over which the equation is coarse-grained (the filter width in the present case) is completely arbitrary (and in practice dictated by the available computational resources), and cannot be justified a priori on physical grounds.

In this paper, we show that a coarse-grained description of hydrodynamics using the microscopic theories is possible. Specifically, we apply the standard filtering procedure (isotropic Gaussian filter) not on the Navier-Stokes equations but on the Boltzmann kinetic equation. We recall that the Navier-Stokes equations are a well defined limit of the Boltzmann equation (the hydrodynamic limit), whereas the filtering operation and going to the hydrodynamic limit are two distinct operations which do not commute, because kinetic fluctuations generally do not annihilate upon filtering. By doing so, we obtain the following results:

- Smallness parameter: The smallness parameter of the present theory is the usual kinetic-theory Knudsen number Kn,

$$
\mathrm{Kn}=\frac{\nu}{L c_{\mathrm{s}}} \sim \frac{\mathrm{Ma}}{\mathrm{Re}}
$$

where Ma is the Mach number and Re is the Reynolds number, $\nu$ is the kinematic viscosity, $c_{\mathrm{s}}$ is the sound speed and $L$ is the characteristic macroscopic length. Smallness of Kn rules emergence of both, the usual viscosity terms, and the subgrid contributions, on the viscosity time scale of the filtered Boltzmann equation (that is, in the first-order Chapman-Enskog solution to the filtered Boltzmann equation).

- Scaling: In the coarse-grained representation obtained by filtering, the filterwidth $\Delta$ (for the Gaussian filter, $\Delta^{2}$ is proportional to the covariance) is the smallest length-scale to be resolved. The requirement that contributions from the subgrid scales appear in the kinetic picture at the time scale of molecular relaxation time (viscosity time scale) sets the scaling of $\Delta$ with the Knudsen number as follows:

$$
\Delta=k L \sqrt{\mathrm{Kn}}
$$

where $k$ is a nonuniversal constant which scales neither with $L$, nor with Kn. For the sake of simplicity, we set $k=1$ in all the further computations. Equations (1) and (2) imply that the filter-width scales with the Reynolds number as follows:

$$
\Delta \sim \operatorname{Re}^{-1 / 2}
$$


While the Kolmogorov length, $l_{\mathrm{K}}$, scales as $l_{\mathrm{K}} \sim \mathrm{Re}^{-3 / 4}$, we have

$$
\frac{\Delta}{l_{\mathrm{K}}} \sim \operatorname{Re}^{1 / 4}
$$

Thus, the filtering scale is larger than the Kolmogorov scale when Re is large enough.

- Subgrid model: With the above smallness parameter (1), and the scaling (2), we rigorously derive the following subgrid pressure tensor $P_{\alpha \beta}^{\mathrm{SG}}$, in addition to the usual (advection and viscosity) terms in the momentum equation:

$$
\begin{aligned}
P_{\alpha \beta}^{\mathrm{SG}} & =\frac{\operatorname{Kn} L^{2} \bar{\rho}}{12}\left[\bar{S}_{\alpha \gamma}-\bar{\Omega}_{\alpha \gamma}\right]\left[\bar{S}_{\gamma \beta}+\bar{\Omega}_{\gamma \beta}\right] \\
& =\frac{\nu \bar{\rho} c_{\mathrm{S}} L}{12}\left[\bar{S}_{\alpha \gamma}-\bar{\Omega}_{\alpha \gamma}\right]\left[\bar{S}_{\gamma \beta}+\bar{\Omega}_{\gamma \beta}\right] .
\end{aligned}
$$

Here $\bar{\rho}$ is the filtered density, and summation convention in spatial components is adopted. For any function $X, \bar{X}$ denotes the filtered value of $X$. Furthermore, the filtered rate of the strain tensor $\bar{S}_{\alpha \beta}$ and the filtered rate of the rotation tensor $\bar{\Omega}_{\alpha \beta}$ depends only the large scale velocity, $\overline{u_{\alpha}}$ :

$$
\begin{aligned}
& \bar{\Omega}_{\alpha \beta}=\frac{1}{2}\left\{\partial_{\alpha} \overline{u_{\beta}}-\partial_{\beta} \overline{u_{\alpha}}\right\}, \\
& \bar{S}_{\alpha \beta}=\frac{1}{2}\left\{\partial_{\alpha} \overline{u_{\beta}}+\partial_{\beta} \overline{u_{\alpha}}\right\} .
\end{aligned}
$$

The derived subgrid model belongs to the class of Smagorinsky models [4], and the tensorial structure of the subgrid pressure tensor (5) corresponds to the so-called tensor-diffusivity subgrid model (TDSG) introduced by Leonard [5], and which became popular after the work of Clark et al [6]. Here, it is interesting to recall that in the class of existing Smagorinsky models the TDSG is one of only a few models in which the sub-grid scale stress tensor remains frame-invariant under arbitrary time-dependent rotations of reference frame [7]. Furthermore, the TDSG model belongs to a subclass of Smagorinsky models which take into account the backscattering of energy from the small scale to the large scales [1]. Beginning with the seminal work of Kraichnan [8], importance of the backscattering of energy in turbulence modeling is commonly recognized.

- Uniqueness: The result (5) requires only isotropy of the filter but otherwise is independent of the particular functional form of the filter. There are no other subgrid models different from (5) which can be derived from kinetic theory by the one-step filtering procedure. In other words, higher-order spatial derivatives are not neglected in an uncontrolled fashion, rather, they are of the order $\mathrm{Kn}^{2}$, and thus do not show up on the viscosity time scale.

- Nonarbitrary filter-width: Unlike the phenomenological TDSGM where the prefactor in Eq. (5) remains an unspecified " $\Delta$ ", kinetic theory suggests that the filter-width cannot be set at will, rather, it must respect the phys- 
ical parameterization (specific values of Re, Kn etc) of a given setup when the subgrid model is used for numerical simulation. Recent findings that simulations of the TDSG model become unstable for large $\Delta[9]$ is in qualitative agreement with the present result that the filter-width $\Delta$ cannot be made arbitrary large.

The structure of the paper is as follows: In section 2 we set up the kinetic theory for the subsequent coarse-graining and derivation of the subgrid model. It is important to stress that the only requirement on the choice of the kinetic equation in the present context is that it gives the Navier-Stokes hydrodynamic equations in the appropriate fully resolved limit. For that reason we choose to work with a recently introduced minimal kinetic model $[2,3]$ which is sufficient for our present purpose. Filtered kinetic equation is obtained in section 3. In section 4 we derive the subgrid model (5) using the Chapman-Enskog method [10] for the filtered kinetic model. This derivation uniquely defines the scaling (3) from the requirement that the subgrid terms appear on the viscous time scale. Finally, results are summarized, and some directions of future research are discussed in section 6 .

\section{Kinetic theory}

For the present discussion, the particular choice of the kinetic model is unimportant as long as the hydrodynamic limit of the kinetic theory is the usual Navier-Stokes equations at least up to the order $O\left(\mathrm{Ma}^{3}\right)$. We demonstrate the whole procedure in detail for a recently introduced minimal discrete-velocity kinetic model $[2,3]$. As the final result is just the same in two and three dimensional cases, for the sake of simplicity we chose to demonstrate the whole procedure using the two-dimensional model $(D=2)$. The kinetic equation is,

$$
\partial_{t} f_{i}+C_{i \alpha} \partial_{\alpha} f_{i}=-\tau^{-1}\left(f_{i}-f_{i}^{\mathrm{eq}}\right),
$$

where $f_{i}(\mathbf{x}, t), i=1, \ldots 9$ are populations of discrete velocities $\mathbf{C}_{i}$ :

$$
\begin{aligned}
& C_{x}=\{0,1,0,-1,0,1,-1,-1,1\}, \\
& C_{y}=\{0,0,1,0,-1,1,1,-1,-1\} .
\end{aligned}
$$

The local equilibrium $f_{i}^{\text {eq }}$ is the conditional minimizer of the the entropy function $H$ :

$$
H=\sum_{i=1}^{9} f_{i} \ln \left(\frac{f_{i}}{W_{i}}\right),
$$


under the constraint of local conservation laws:

$$
\sum_{i=1}^{9} f_{i}^{\mathrm{eq}}\left\{1, \mathbf{C}_{i}\right\} .=\{\rho, \rho \mathbf{u}\}
$$

The weights $W_{i}$ in the equation (10) are:

$$
W=\frac{1}{36}\{16,4,4,4,4,1,1,1,1\} .
$$

The explicit expression for $f_{i}^{\text {eq }}$ reads:

$$
f_{i}^{\mathrm{eq}}=\rho W_{i} \prod_{\alpha=1}^{D}\left(2-\sqrt{1+3 u_{\alpha}^{2}}\right)\left(\frac{2 u_{\alpha}+\sqrt{1+3 u_{\alpha}^{2}}}{1-u_{\alpha}}\right)^{C_{i \alpha}} .
$$

Below, it will prove convenient to work in the moment representation rather than in the population representation. Let us choose the following orthogonal set of basis vectors in the 9-dimensional phase space of the kinetic equation $(7)$ :

$$
\begin{aligned}
& \psi_{1}=\{1,1,1,1,1,1,1,1,1\}, \\
& \psi_{2}=\{0,1,0,-1,0,1,-1,-1,1\}, \\
& \psi_{3}=\{0,0,1,0,-1,1,1,-1,-1\}, \\
& \psi_{4}=\{0,0,0,0,0,1,-1,1,-1\}, \\
& \psi_{5}=\{0,1,-1,1,-1,0,0,0,0\}, \\
& \psi_{6}=\{0,-2,0,2,0,1,-1,-1,1\}, \\
& \psi_{7}=\{0,0,-2,0,2,1,1,-1,-1\}, \\
& \psi_{8}=\{4,-5,-5,-5,-5,4,4,4,4\}, \\
& \psi_{9}=\{4,0,0,0,0,-1,-1,-1,-1\} .
\end{aligned}
$$

The orthogonality of the chosen basis is in the sense of the usual Euclidean scalar product, i.e.,

$$
\sum_{k=1}^{9} \psi_{i k} \psi_{k j}=d_{i} \delta_{i j}
$$

where $d_{i}$ are some constants needed for the normalization (the basis vectors are orthogonal but not orthonormal). We define new variables $M_{i}, i=1, \ldots, 9$ as:

$$
M_{i}=\sum_{j=1}^{9} \psi_{i j} f_{j}
$$

where $\psi_{i j}$ denotes $j$ th component of the 9-dimensional vector $\psi_{i}$. Basic hydrodynamic fields are $M_{1}=\rho, M_{2}=\rho u_{x}$, and $M_{3}=\rho u_{y}$. The remaining six moments are related to higher order moments of the distribution (the pressure tensor $P_{\alpha \beta}=\sum f_{i} C_{i \alpha} C_{i \beta}$ and the third order moment $Q_{\alpha \beta \gamma}=\sum f_{i} C_{i \alpha} C_{i \beta} C_{i \gamma}$ and so on), as: $M_{4}=P_{x y}$, 


$$
\begin{aligned}
& M_{5}=P_{x x}-P_{y y}, \\
& M_{6}=3 \sum f_{i} C_{i y}^{2} C_{i x}-2 M_{2}, \\
& M_{7}=3 \sum f_{i} C_{i x}^{2} C_{i y}-2 M_{3}
\end{aligned}
$$

The explicit form of the stress tensor in term of the new set of variables is:

$$
\begin{aligned}
P_{x y} & =M_{4}, \\
P_{x x} & =\frac{2}{3} M_{1}+\frac{1}{2} M_{5}+\frac{1}{30} M_{8}-\frac{1}{5} M_{9}, \\
P_{y y} & =\frac{2}{3} M_{1}-\frac{1}{2} M_{5}+\frac{1}{30} M_{8}-\frac{1}{5} M_{9} .
\end{aligned}
$$

The time evolution equations for the set of moments are:

$$
\begin{aligned}
\partial_{t} M_{1}+\partial_{x} M_{2}+\partial_{y} M_{3} & =0 \\
\partial_{t} M_{2}+\partial_{x}\left(\frac{2}{3} M_{1}+\frac{1}{2} M_{5}+\frac{1}{30} M_{8}-\frac{1}{5} M_{9}\right)+\partial_{y} M_{4} & =0 \\
\partial_{t} M_{3}+\partial_{x} M_{4}+\partial_{y}\left(\frac{2}{3} M_{1}-\frac{1}{2} M_{5}+\frac{1}{30} M_{8}-\frac{1}{5} M_{9}\right) & =0 \\
\partial_{t} M_{4}+\frac{1}{3} \partial_{x}\left(2 M_{3}+M_{7}\right)+\frac{1}{3} \partial_{y}\left(2 M_{2}+M_{6}\right) & =\frac{1}{\tau}\left(M_{4}^{\mathrm{eq}}\left(M_{1}, M_{2}, M_{3}\right)-M_{4}\right), \\
\partial_{t} M_{5}+\frac{1}{3} \partial_{x}\left(M_{2}-M_{6}\right)+\frac{1}{3} \partial_{y}\left(M_{7}-M_{3}\right) & \left.=\frac{1}{\tau}\left(M_{5}^{\mathrm{eq}}\left(M_{1}, M_{2}, M_{3}\right)-M_{(51}\right)\right) \\
\partial_{t} M_{6}-\frac{1}{5} \partial_{x}\left(5 M_{5}-M_{8}+M_{9}\right)+\partial_{y} M_{4} & =\frac{1}{\tau}\left(M_{6}^{\mathrm{eq}}\left(M_{1}, M_{2}, M_{3}\right)-M_{6}\right), \\
\partial_{t} M_{7}+\partial_{x} M_{4}+\frac{1}{5} \partial_{y}\left(5 M_{5}+M_{8}-M_{9}\right) & =\frac{1}{\tau}\left(M_{7}^{\mathrm{eq}}\left(M_{1}, M_{2}, M_{3}\right)-M_{7}\right), \\
\partial_{t} M_{8}+\partial_{x}\left(M_{2}+3 M_{6}\right)+\partial_{y}\left(M_{3}+3 M_{7}\right) & =\frac{1}{\tau}\left(M_{8}^{\mathrm{eq}}\left(M_{1}, M_{2}, M_{3}\right)-M_{8}\right), \\
\partial_{t} M_{9}-\frac{1}{3} \partial_{x}\left(2 M_{2}+M_{6}\right)-\frac{1}{3} \partial_{y}\left(2 M_{3}+M_{7}\right) & =\frac{1}{\tau}\left(M_{9}^{\mathrm{eq}}\left(M_{1}, M_{2}, M_{3}\right)-M_{9}\right) .
\end{aligned}
$$

The expression for the local equilibrium moments $M_{i}^{\mathrm{eq}}, i=4, \ldots, 9$ in terms of the basic variables $M_{1}, M_{2}$, and $M_{3}$ to the order $u^{2}$ is: 


$$
\begin{aligned}
& M_{4}^{\mathrm{eq}}\left(M_{1}, M_{2}, M_{3}\right)=\frac{M_{2} M_{3}}{M_{1}}, \\
& M_{5}^{\mathrm{eq}}\left(M_{1}, M_{2}, M_{3}\right)=\frac{M_{2}^{2}-M_{3}^{2}}{M_{1}}, \\
& M_{6}^{\mathrm{eq}}\left(M_{1}, M_{2}, M_{3}\right)=-M_{2}, \\
& M_{7}^{\mathrm{eq}}\left(M_{1}, M_{2}, M_{3}\right)=-M_{3}, \\
& M_{8}^{\mathrm{eq}}\left(M_{1}, M_{2}, M_{3}\right)=-3 \frac{M_{2}^{2}+M_{3}^{2}}{M_{1}}, \\
& M_{9}^{\mathrm{eq}}\left(M_{1}, M_{2}, M_{3}\right)=\frac{5}{3} M_{1}-\frac{3\left(M_{2}^{2}+M_{3}^{2}\right)}{M_{1}} .
\end{aligned}
$$

The incompressible Navier-Stokes equations are the hydrodynamic limit of the system (19) and (20).

In the next section, we shall remove small scales through a filtering procedure on the moment system (19), (20). A precise definition of the small scales is postponed until later sections. For the time being, let us assume that there exist a length-scale $\Delta$, and we wish to look at the hydrodynamics at lengthscale larger than $\Delta$ only.

\section{$3 \quad$ Filtered kinetic theory}

Coarse-grained versions of the Boltzmann equations have been discussed in the recent literature $[11,12,13]$. However, a systematic treatment is still lacking. In this section, we shall fill this gap.

\subsection{Gaussian filter}

For any function $X$, the filtered function $\bar{X}$ is defined as:

$$
\bar{X}(\mathbf{x})=\int_{R^{D}} G(\mathbf{r}) X(\mathbf{x}-\mathbf{r}) d \mathbf{r} .
$$

Function $G$ is called the filter. In the sequel, we apply the filtering operation (21) on the moment system (19). We will need two relations. First, for any function $X$,

$$
\overline{\partial_{\alpha} X}=\partial_{\alpha} \bar{X}
$$

This relation is sufficient to filter the propagation terms in the equation (19) due to linearity of propagation in the kinetic picture. The latter is a useful 
property which is not shared by the hydrodynamic Navier-Stokes equations, where the nonlinearity and nonlocality both come into the same $(\boldsymbol{u} \nabla \boldsymbol{u})$ term. Any isotropic filter, which satisfies the condition of commuting of the derivatives under the application of the filter (Eq. 22), will suffice for the present purpose. We choose a standard Gaussian filter [1] which has the property (22):

$$
G(\mathbf{r}, \Delta)=\left(\frac{6}{\pi \Delta^{2}}\right)^{D} \exp \left(-\frac{6 \mathbf{r}^{2}}{\Delta^{2}}\right)
$$

Let us recall the isotropy properties of a Gaussian filter:

$$
\begin{gathered}
\int_{R^{D}} G(\mathbf{r}, \Delta) d \mathbf{r}=1 \\
\int_{R^{D}} G(\mathbf{r}, \Delta) \mathbf{r} d \mathbf{r}=0 \\
\int_{R^{D}} G(\mathbf{r}, \Delta) r_{\alpha} r_{\beta} d \mathbf{r}=\frac{\Delta^{2}}{12} \delta_{\alpha \beta} .
\end{gathered}
$$

Second, in order to filter the nonlinear terms (20) in the right hand side of moment equations (19), we will also need the following relation for three arbitrary functions $X, Y, Z$ which follow immediately from the isotropy property by second-order Taylor expansion:

$$
\begin{aligned}
\overline{\left(\frac{X Y}{Z}\right)} & =\left(\frac{\bar{X} \bar{Y}}{\bar{Z}}\right)+\frac{\Delta^{2}}{12 \bar{Z}}\left\{\left(\partial_{\alpha} \bar{X}\right)\left(\partial_{\alpha} \bar{Y}\right)-\frac{2}{\bar{Z}}\left(\partial_{\alpha} \bar{Z}\right)\left(\bar{X} \partial_{\alpha} \bar{Y}+\bar{Y} \partial_{\alpha} \bar{X}+\frac{2 \overline{X Y}}{\bar{Z}} \partial_{\alpha} \bar{Z}\right)\right\} \\
& +O\left(\Delta^{4}\right) .
\end{aligned}
$$

The effect of a Gaussian filter need not be truncated to any order at the present step. The higher-order terms lumped under $O\left(\Delta^{4}\right)$ in equation (25) can be computed from elementary Gaussian integrals. As we shall see it soon, higher than second order terms disappear in the hydrodynamic limit once the scaling of the filter-width versus Knudsen number is appropriately chosen.

In the next section, we shall filter the moment equations (19).

\subsection{Filtering the moment system}

Applying the filter (21) to the moment system (19), (20), using (22) and (25), and keeping terms up to the order $u^{2}$, we obtain the following filtered moment system: 


$$
\begin{aligned}
& \partial_{t} \bar{M}_{1}+\partial_{x} \bar{M}_{2}+\partial_{y} \bar{M}_{3}=0 \\
& \partial_{t} \bar{M}_{2}+\partial_{x}\left(\frac{2}{3} \bar{M}_{1}+\frac{1}{2} \bar{M}_{5}+\frac{1}{30} \bar{M}_{8}-\frac{1}{5} \bar{M}_{9}\right)+\partial_{y} \bar{M}_{4}=0, \\
& \partial_{t} \bar{M}_{3}+\partial_{x} \bar{M}_{4}+\partial_{y}\left(\frac{2}{3} \bar{M}_{1}-\frac{1}{2} \bar{M}_{5}+\frac{1}{30} \bar{M}_{8}-\frac{1}{5} \bar{M}_{9}\right)=0 \\
& \partial_{t} \bar{M}_{4}+\frac{1}{3} \partial_{x}\left(2 \bar{M}_{3}+\bar{M}_{7}\right)+\frac{1}{3} \partial_{y}\left(2 \bar{M}_{2}+\bar{M}_{6}\right)=\frac{1}{\tau}\left(M_{4}^{\mathrm{eq}}\left(\bar{M}_{1}, \bar{M}_{2}, \bar{M}_{3}\right)-\bar{M}_{4}\right) \\
& +\frac{\Delta^{2}}{12 \tau \bar{M}_{1}}\left(\partial_{\alpha} \bar{M}_{2}\right)\left(\partial_{\alpha} \bar{M}_{3}\right)+O\left(\frac{\Delta^{4}}{\tau}\right) \text {, } \\
& \partial_{t} \bar{M}_{5}+\frac{1}{3} \partial_{x}\left(\bar{M}_{2}-\bar{M}_{6}\right)+\frac{1}{3} \partial_{y}\left(\bar{M}_{7}-\bar{M}_{3}\right)=\frac{1}{\tau}\left(M_{5}^{\mathrm{eq}}\left(\bar{M}_{1}, \bar{M}_{2}, \bar{M}_{3}\right)-\bar{M}_{5}\right) \\
& +\frac{\Delta^{2}}{12 \tau \bar{M}_{1}}\left\{\left(\partial_{\alpha} \bar{M}_{2}\right)\left(\partial_{\alpha} \bar{M}_{2}\right)-\left(\partial_{\alpha} \bar{M}_{3}\right)\left(\partial_{\alpha} \bar{M}_{3}\right)\right\}+O\left(\frac{\Delta^{4}}{\tau}\right), \\
& \partial_{t} \bar{M}_{6}-\frac{1}{5} \partial_{x}\left(5 \bar{M}_{5}-\bar{M}_{8}+\bar{M}_{9}\right)+\partial_{y} \bar{M}_{4}=\frac{1}{\tau}\left(M_{6}^{\mathrm{eq}}\left(\bar{M}_{1}, \bar{M}_{2}, \bar{M}_{3}\right)-\bar{M}_{6}\right) \\
& \partial_{t} \bar{M}_{7}+\partial_{x} \bar{M}_{4}+\frac{1}{5} \partial_{y}\left(5 \bar{M}_{5}+\bar{M}_{8}-\bar{M}_{9}\right)=\frac{1}{\tau}\left(M_{7}^{\mathrm{eq}}\left(\bar{M}_{1}, \bar{M}_{2}, \bar{M}_{3}\right)-\bar{M}_{7}\right), \\
& \partial_{t} \bar{M}_{8}+\partial_{x}\left(\bar{M}_{2}+3 \bar{M}_{6}\right)+\partial_{y}\left(\bar{M}_{3}+3 \bar{M}_{7}\right)=\frac{1}{\tau}\left(M_{8}^{\mathrm{eq}}\left(\bar{M}_{1}, \bar{M}_{2}, \bar{M}_{3}\right)-\bar{M}_{8}\right) \\
& -\frac{3 \Delta^{2}}{12 \tau \bar{M}_{1}}\left\{\left(\partial_{\alpha} \bar{M}_{2}\right)\left(\partial_{\alpha} \bar{M}_{2}\right)+\left(\partial_{\alpha} \bar{M}_{3}\right)\left(\partial_{\alpha} \bar{M}_{3}\right)\right\}+O\left(\frac{\Delta^{4}}{\tau}\right) \text {, } \\
& \partial_{t} \bar{M}_{9}-\frac{1}{3} \partial_{x}\left(2 \bar{M}_{2}+\bar{M}_{6}\right)-\frac{1}{3} \partial_{y}\left(2 \bar{M}_{3}+\bar{M}_{7}\right)=\frac{1}{\tau}\left(M_{9}^{\mathrm{eq}}\left(\bar{M}_{1}, \bar{M}_{2}, \bar{M}_{3}\right)-\bar{M}_{9}\right) \\
& -\frac{3 \Delta^{2}}{12 \tau \bar{M}_{1}}\left\{\left(\partial_{\alpha} \bar{M}_{2}\right)\left(\partial_{\alpha} \bar{M}_{2}\right)+\left(\partial_{\alpha} \bar{M}_{3}\right)\left(\partial_{\alpha} \bar{M}_{3}\right)\right\}+O\left(\frac{\Delta^{4}}{\tau}\right) \text {. }
\end{aligned}
$$

Thus, we are set up to derive the hydrodynamic equations as the appropriate limit of the filtered kinetic system (26). In passing, we note that different moments relax with different effective relaxation time scales, because the subgrid terms are not the same for all kinetic moments.

\section{Hydrodynamic limit of the filtered kinetic theory}

In the kinetic equation we have a natural length scale set by Knudsen number Kn (1). The Navier-Stokes dynamics is obtained in the limit $\mathrm{Kn} \ll 1$. By filtering the kinetic equation we have introduced a new length scale as the size of the filter $\Delta$. The hydrodynamic equations produced by the filtered kinetic equation will depend on how $\Delta$ scales with the Knudsen number. In order to understand this issue, let us look at the filtered equation for one of

the moments (26) in the non-dimensional form. In order to do this, let us 
introduce scaled time and space variables,

$$
\begin{aligned}
\mathbf{x}^{\prime} & =\frac{\mathbf{x}}{L}, \\
t^{\prime} & =\frac{t c_{\mathrm{s}}}{L},
\end{aligned}
$$

where $c_{\mathrm{s}}=1 / \sqrt{3}$ for the present model. Let us also specify Knudsen number in terms of the relaxation time $\tau$ :

$$
\mathrm{Kn}=\frac{\nu}{L c_{\mathrm{s}}} \equiv \frac{\tau c_{\mathrm{s}}}{L}
$$

where, $\nu$ is the kinematic viscosity, $\nu=\tau c_{\mathrm{s}}^{2}$ in the present model. Then, for example, the filtered equation for the moment $M_{4}$ reads:

$$
\begin{aligned}
\partial_{t^{\prime}} \bar{M}_{4} & +\frac{1}{3 c_{s}} \partial_{x^{\prime}}\left(2 \bar{M}_{3}+\bar{M}_{7}\right)+\frac{1}{3 c_{s}} \partial_{y^{\prime}}\left(2 \bar{M}_{2}+\bar{M}_{6}\right)=\frac{1}{\mathrm{Kn}}\left(M_{4}^{\mathrm{eq}}\left(\bar{M}_{1}, \bar{M}_{2}, \bar{M}_{3}\right)-\bar{M}_{4}\right) \\
& +\frac{\Delta^{2}}{12 \mathrm{Kn} L^{2} \bar{M}_{1}}\left\{\left(\partial_{x^{\prime}} \bar{M}_{2}\right)\left(\partial_{x^{\prime}} \bar{M}_{3}\right)+\left(\partial_{y^{\prime}} \bar{M}_{2}\right)\left(\partial_{y^{\prime}} \bar{M}_{3}\right)\right\}+O\left(\frac{\Delta^{4}}{L^{4} \mathrm{Kn}}\right) .
\end{aligned}
$$

We see that the in absence of the filter $(\Delta=0)$, the usual situation of a singularly perturbed kinetic equation is recovered (and this results in the Navier-Stokes equations in the first-order Chapman-Enskog expansion). Let us consider the following three possibilities of dependence of $\Delta$ on $\mathrm{Kn}$ :

- If $\Delta / L \sim \mathrm{Kn}^{0}$, then we do not have a singularly perturbed equation in (29) anymore. That is, the filter is too wide, and it affects the advection terms in the hydrodynamic equations.

- If $\Delta / L \sim \mathrm{Kn}$, then we do have a singularly perturbed system. However, the subgrid terms are of order $\mathrm{Kn}^{2}$, and they do not show up in the order Kn hydrodynamic equation. In other words, the filter is too narrow so that it does not affect hydrodynamic equations at the viscous time scale.

- Finally, there is only one possibility to set the scaling of filter-width with Kn so that the system is singularly perturbed, and the subgrid terms of the order $\Delta^{2}$ contribute just at the viscous time scale. This situation happens if

$$
\frac{\Delta}{L} \sim \sqrt{\mathrm{Kn}}
$$

Note that, with the scaling (30), all the higher-order terms (of the order $\Delta^{4}$ and higher) become of the order $\mathrm{Kn}$ and higher, so that they do not contribute at the viscous time scale.

Once the scaling of the filter-width (30) is introduced into the filtered moment equations (26), the application of the Chapman-Enskog method [10] becomes 
a routine. We write:

$$
\partial_{t}=\partial_{t}^{(0)}+\mathrm{Kn} \partial_{t}^{(1)}+O\left(\mathrm{Kn}^{2}\right)
$$

and for $i=4, \ldots, 9$ :

$$
\bar{M}_{i}=M_{i}^{\mathrm{eq}}\left(\bar{M}_{1}, \bar{M}_{2}, \bar{M}_{3}\right)+\mathrm{Kn} \bar{M}_{i}^{(1)}+O\left(\mathrm{Kn}^{2}\right) .
$$

The hydrodynamics equations at the the order $O(1)$ are the Euler equations:

$$
\begin{aligned}
\partial_{t}^{(0)} \bar{M}_{1} & =-\partial_{x} \bar{M}_{2}-\partial_{y} \bar{M}_{3} \\
\partial_{t}^{(0)} \bar{M}_{2} & =-\partial_{x}\left(\bar{M}_{1} c_{s}^{2}+\frac{\bar{M}_{2} \bar{M}_{2}}{\bar{M}_{1}}\right)-\partial_{y}\left(\frac{\bar{M}_{2} \bar{M}_{3}}{\bar{M}_{1}}\right), \\
\partial_{t}^{(0)} \bar{M}_{3} & =-\partial_{x}\left(\frac{\bar{M}_{2} \bar{M}_{3}}{\bar{M}_{1}}\right)-\partial_{y}\left(\bar{M}_{1} c_{s}^{2}+\frac{\bar{M}_{3} \bar{M}_{3}}{\bar{M}_{1}}\right) .
\end{aligned}
$$

Note that no subgrid terms appear at this time scale in the hydrodynamic equations (33). This means that large scale motion, even after filtering, is dictated just by the conservation laws. Zero-order time derivatives of the nonconserved moments are evaluated using the chain rule:

$$
\partial_{t}^{(0)} M_{i}^{\mathrm{eq}}\left(\bar{M}_{1}, \bar{M}_{2}, \bar{M}_{3}\right)=\frac{\partial M_{i}^{\mathrm{eq}}}{\partial \bar{M}_{1}} \partial_{t}^{(0)} \bar{M}_{1}+\frac{\partial M_{i}^{\mathrm{eq}}}{\partial \bar{M}_{2}} \partial_{t}^{(0)} \bar{M}_{2}+\frac{\partial M_{i}^{\mathrm{eq}}}{\partial \bar{M}_{3}} \partial_{t}^{(0)} \bar{M}_{3} .
$$

In particular, to the order $u^{2}$ :

$$
\begin{aligned}
& \partial_{t}^{(0)} M_{4}^{\mathrm{eq}}\left(\bar{M}_{1}, \bar{M}_{2}, \bar{M}_{3}\right)=0 \\
& \partial_{t}^{(0)} M_{5}^{\mathrm{eq}}\left(\bar{M}_{1}, \bar{M}_{2}, \bar{M}_{3}\right)=0 \\
& \partial_{t}^{(0)} M_{6}^{\mathrm{eq}}\left(\bar{M}_{1}, \bar{M}_{2}, \bar{M}_{3}\right)=\partial_{x}\left(\bar{M}_{1} c_{s}^{2}+\frac{\bar{M}_{2} \bar{M}_{2}}{\bar{M}_{1}}\right)+\partial_{y}\left(\frac{\bar{M}_{2} \bar{M}_{3}}{\bar{M}_{1}}\right), \\
& \partial_{t}^{(0)} M_{7}^{\mathrm{eq}}\left(\bar{M}_{1}, \bar{M}_{2}, \bar{M}_{3}\right)=\partial_{x}\left(\frac{\bar{M}_{2} \bar{M}_{3}}{\bar{M}_{1}}\right)+\partial_{y}\left(\bar{M}_{1} c_{s}^{2}+\frac{\bar{M}_{3}}{\bar{M}_{1}}\right), \\
& \partial_{t}^{(0)} M_{8}^{\mathrm{eq}}\left(\bar{M}_{1}, \bar{M}_{2}, \bar{M}_{3}\right)=0, \\
& \partial_{t}^{(0)} M_{9}^{\mathrm{eq}}\left(\bar{M}_{1}, \bar{M}_{2}, \bar{M}_{3}\right)=-\frac{5}{3}\left[\partial_{x} \bar{M}_{2}+\partial_{y} \bar{M}_{3}\right] .
\end{aligned}
$$

At the next order $O(\mathrm{Kn})$, correction to locally conserved moments is equal to zero,

$$
\bar{M}_{1}^{(1)}=\bar{M}_{2}^{(1)}=\bar{M}_{3}^{(1)}=0
$$


whereas corrections to the non-conserved moments, $\bar{M}_{i}^{(1)}, i=4, \ldots, 9$, are obtained by substituting Eq. (31), and Eq. (32) in Eq. (26) and eliminating the zeroth order time derivatives using Eq. (35):

$$
\begin{aligned}
& \bar{M}_{4}^{(1)}=-L c_{s}\left[\partial_{x} \bar{M}_{3}+\partial_{y} \bar{M}_{2}\right]+\frac{L^{2}}{12 \bar{M}_{1}}\left\{\left(\partial_{x} \bar{M}_{2}\right)\left(\partial_{x} \bar{M}_{3}\right)+\left(\partial_{y} \bar{M}_{2}\right)\left(\partial_{y} \bar{M}_{3}\right)\right\} \\
& \bar{M}_{5}^{(1)}=-2 L c_{s}\left[\partial_{x} \bar{M}_{2}-\partial_{y} \bar{M}_{3}\right]+\frac{L^{2}}{12 \bar{M}_{1}}\left\{\left(\partial_{x} \bar{M}_{2}\right)^{2}-\left(\partial_{x} \bar{M}_{3}\right)^{2}+\left(\partial_{y} \bar{M}_{2}\right)^{2}-\left(\partial_{y} \bar{M}_{3}\right)^{2}\right\} \\
& \bar{M}_{6}^{(1)}=-L c_{s}\left[\partial_{x}\left\{\frac{3\left(\bar{M}_{3}^{2}\right)}{\bar{M}_{1}}\right\}+\partial_{y} \frac{6 \bar{M}_{2} \bar{M}_{3}}{\bar{M}_{1}}\right], \\
& \bar{M}_{7}^{(1)}=-L c_{s}\left[\partial_{x} \frac{6 \bar{M}_{2} \bar{M}_{3}}{\bar{M}_{1}}+\partial_{y}\left\{\frac{3\left(\bar{M}_{2}^{2}\right)}{\bar{M}_{1}}\right\}\right], \\
& \bar{M}_{8}^{(1)}=6 L c_{s}\left[\partial_{x} \bar{M}_{2}+\partial_{y} \bar{M}_{3}\right]-\frac{3 L^{2}}{12 \bar{M}_{1}}\left\{\left(\partial_{x} \bar{M}_{2}\right)^{2}+\left(\partial_{y} \bar{M}_{2}\right)^{2}+\left(\partial_{x} \bar{M}_{3}\right)^{2}+\left(\partial_{y} \bar{M}_{3}\right)^{2}\right\} \\
& \bar{M}_{9}^{(1)}=6 L c_{s}\left[\partial_{x} \bar{M}_{2}+\partial_{y} \bar{M}_{3}\right]-\frac{3 L^{2}}{12 \bar{M}_{1}}\left\{\left(\partial_{x} \bar{M}_{2}\right)^{2}+\left(\partial_{y} \bar{M}_{2}\right)^{2}+\left(\partial_{x} \bar{M}_{3}\right)^{2}+\left(\partial_{y} \bar{M}_{3}\right)^{2}\right\} .
\end{aligned}
$$

and the first-order time derivative of the conserved moments are:

$$
\begin{aligned}
& \partial_{t}^{(1)} \bar{M}_{1}=0 \\
& \partial_{t}^{(1)} \bar{M}_{2}=-\partial_{x}\left(\frac{1}{2} \bar{M}_{5}^{(1)}+\frac{1}{30} \bar{M}_{8}^{(1)}-\frac{1}{5} \bar{M}_{9}^{(1)}\right)-\partial_{y} M_{4}^{(1)} \\
& \partial_{t}^{(1)} \bar{M}_{3}=-\partial_{x} M_{4}^{(1)}-\partial_{y}\left(-\frac{1}{2} \bar{M}_{5}^{(1)}+\frac{1}{30} \bar{M}_{8}^{(1)}-\frac{1}{5} \bar{M}_{9}^{(1)}\right)
\end{aligned}
$$

These equations shows that the viscous term and the subgrid term both appear as the $O(K n)$ contribution. We remind that no assumption was made about relative magnitude of the subgrid term as compared with the viscous terms. The only requirement that is set on the subgrid scale term is that they appear at the viscous time scale rather than the time scale of the advection. We can write the complete hydrodynamics equation by using Eq. (31), Eq. (32), Eq. (33), Eq. (36), and Eq. (37) to obtain the hydrodynamics equations correct up to the order $O\left(\mathrm{Kn}^{2}\right)$ (at this stage one recovers the Navier-Stokes equations using the unfiltered kinetic equation). In the next section, we shall see how the subgrid scale terms affect the Navier-Stokes description. 


\section{$5 \quad$ Hydrodynamic equations}

The final set of hydrodynamics equation, valid up to the order $O\left(\mathrm{Kn}^{2}\right)$ is:

$$
\begin{array}{r}
\partial_{t} \bar{\rho}+\partial_{x} \overline{\rho u_{x}}+\partial_{y} \overline{\rho u_{y}}=0 \\
\partial_{t} \overline{\rho u_{x}}+\partial_{x} \bar{P}_{x x}+\partial_{y} \bar{P}_{x y}=0 \\
\partial_{t} \overline{\rho u_{y}}+\partial_{x} \bar{P}_{x y}+\partial_{y} \bar{P}_{y y}=0
\end{array}
$$

where

$$
\begin{aligned}
& \bar{P}_{x x}=\left[\bar{P}+\frac{{\overline{\rho u_{x}}}^{2}}{\bar{\rho}}-2 \nu \bar{\rho}_{x x}\right]+P_{x x}^{\mathrm{SG}}, \\
& \bar{P}_{x y}=\left[\frac{\overline{\rho u_{x}} \overline{\rho u_{y}}}{\bar{\rho}}-2 \nu \bar{\rho} \bar{S}_{x y}\right]+P_{x y}^{\mathrm{SG}}, \\
& \bar{P}_{y y}=\left[\bar{P}+\frac{{\overline{\rho u_{y}}}^{2}}{\bar{\rho}}-2 \nu \bar{\rho} \bar{S}_{y y}\right]+P_{y y}^{\mathrm{SG}},
\end{aligned}
$$

with $\bar{P}=\bar{\rho} c_{s}^{2}$, as the thermodynamic pressure and

$$
P_{\alpha \beta}^{\mathrm{SG}}=\frac{\mathrm{Kn} L^{2} \bar{\rho}}{12}\left[\bar{S}_{\alpha \gamma}-\bar{\Omega}_{\alpha \gamma}\right]\left[\bar{S}_{\gamma \beta}+\bar{\Omega}_{\gamma \beta}\right]=\frac{\nu \bar{\rho} L}{12 c_{s}}\left[\bar{S}_{\alpha \gamma}-\bar{\Omega}_{\alpha \gamma}\right]\left[\bar{S}_{\gamma \beta}+\bar{\Omega}_{\gamma \beta}\right]
$$

Thus, we have obtained a closed set of hydrodynamics equations, for appropriate choice of filtering width. This set of equations, written in the nondimensional form up to the order, $u^{2}$ is:

$$
\begin{gathered}
\partial_{\alpha} \overline{u_{\alpha}}=0 \\
\partial_{t}\left(\overline{u_{\alpha}}\right)+\partial_{\beta}\left(\overline{u_{\alpha}} \overline{u_{\beta}}\right)=-\partial_{\alpha} P+2 \operatorname{Kn} \partial_{\beta}\left(\bar{S}_{\alpha \beta}\right) \\
-\frac{\operatorname{Kn}}{12} \partial_{\beta}\left\{\left(\bar{S}_{\alpha \gamma}-\bar{\Omega}_{\alpha \gamma}\right)\left(\bar{S}_{\gamma \beta}+\bar{\Omega}_{\gamma \beta}\right)\right\} .
\end{gathered}
$$

Note that the pressure appearing in the momentum equation is not the thermodynamic pressure anymore, but needs to be computed from the incompressibility condition (Eq. 40) [15]. Significance of the Knudsen number appearing in the equation (41) is explained below in section 6 .

Similar to the case of the Navier-Stokes equation, the subgrid model (5) enjoys the consistent derivation from the kinetic theory. We should remind here again 
that the result is independent of the particular kinetic model used for the derivation. Any kinetic model which recovers the Navier-Stokes equations in the hydrodynamic limit will lead to the same result.

\section{Discussion and conclusion}

Now we shall summarise the results obtained in the present work and their limitations:

- It is possible to derive rigorously a coarse-grained closed set of equation for hydrodynamics, a long cherished goal in turbulence modeling.

- The scale-separation present in the kinetic theory provides a natural way to obtain coarse models.

- Arbitrary choice of filter-width is not allowed.

- In this work, we have shown that the operation of solving the Boltzmann equation (Chapman-Enskog expansion) and coarse-graining (filtering) do not commute. In the usual procedure of producing filtered hydrodynamic equation, the filtering is done on the solution of the Boltzmann equation (Navier-Stokes equations), which leads to closure problems. On the other hand, reversing the order of these two operations, provides a physical meaning to the filtering width and produces a closed set of equations in the hydrodynamic limit.

- As the smallest length scale needed to be resolved in the new set of equations is $\Delta \sim \mathrm{Kn}^{1 / 2} \sim \mathrm{Re}^{-1 / 2}$, the cost of numerical computation reduces drastically as compared to the fully resolved simulation of the Navier-Stokes equations. We can get an estimate of this gain as follows: The smallest scale needed to be resolved in the numerical simulation is proportional to the $\mathrm{Re}^{-1 / 2}$ rather than $\mathrm{Re}^{-3 / 4}$ (Kolmogorov scale). This changes the scaling of number of degrees of freedom in a three-dimensional simulation with Re from $\operatorname{Re}^{9 / 4}$ to $\operatorname{Re}^{3 / 2}$ (number of grid point is $\propto \delta x^{-3}$, where $\delta x$ is the grid spacing). Further, a rough estimate of the scaling of the cost of time integration with $\operatorname{Re}$ is $\operatorname{Re}^{3 / 4}$ (number of time steps is $\propto \delta x^{-1}$ ) in the case of fully resolved simulation of the Navier-Stokes equations [1]. However, in the present case this scaling will be $\mathrm{Ma}^{-1}$. This happens because any numerical scheme has to take a time-step dictated by the sound speed (or an analog of it pertinent to the discretization in time chosen). Thus the scaling of the total cost of computation with the Re changes from $\mathrm{Re}^{3}$ for the Navier-Stokes equations to $\mathrm{Re}^{3 / 2}$ for the present equation.

- In the above estimation, the Mach number Ma appearing in the equation for the filter-width $\Delta\left(\Delta^{2} \propto \mathrm{Re}^{-1}\right)$ was not taken into the account. This is justified as long as Re is sufficiently large and the Mach number $\mathrm{Ma}$ is not zero. An acceptable limit for the incompressible limit of the NavierStokes equation is $\mathrm{Ma} \sim 0.1$ (for example, most of the lattice-Boltzmann 
simulations of the incompressible Navier-Stokes uses Ma $\sim 0.05-0.1$ ). Let us consider the case when the number of grid points in each direction is 1024, then a fully resolved simulation of the Navier-Stokes equations is possible by taking the Reynolds number as Re $\sim O\left(10^{4}\right)$, while a fully resolved simulation using the present subgrid model is possible by taking the Reynolds number as $\operatorname{Re} \sim O\left(10^{5}\right)$, for $\mathrm{Ma} \sim 0.07$.

- One interpretation of the subgrid scale terms is that the removal of the small-scales in the kinetic picture appears naturally as the force term. As the extra terms appearing in the evolution equations for non-conserved variables can also be generated in the unfiltered kinetic equation by an appropriate choice of the external force field (dependent on the position as well as molecular velocity). Thus at least formally, we can find a force-field which will act like a filter and remove the small scales of motion present in the kinetic equation. Thus the physical meaning of the filtering (a purely mathematical operation) at the kinetic level is the application of some self-consistent mean-field force which removes the small scale of the motions from the kinetic equation. The technical advantage of the search for a mean-field force (appearing in the filtered kinetic equation), rather than an effective viscosity term (attempts to absorb subgrid scale contributions in the viscous term of the Navier-Stokes equations) is that the one does not have to deal with the difficult question of what to do with the nonlinearity and nonlocality present in the convective term of the Navier-Stokes equations.

Finally, let us mention some further possible directions of study:

- From a practical standpoint, a major goal of going beyond a Navier-Stokesbased coarse graining, is to make a (filtered) kinetic theory work at possibly large ratios $\Delta / L$. Indeed, successful kinetic subgrid models have been known empirically for some time [14]. However, it is unclear why kinetic subgrid scale models work better than models of the Navier-Stokes equations (for example, a recent comment on the kinetic models of turbulence was: "Whether the approach can be supported by rigorous theory remains to be shown" [16]). Our analysis shows that everything is eventually ruled by the smallness of the Knudsen number, a well defined smallness parameter present in the kinetic theory. This is the first rigorous step in the kinetic modeling of the turbulence. For example, the choice of the filter-width (2), based on the integer-power (standard) Chapman-Enskog analysis is a conservative estimate only. In order to achieve a subgrid model between the advection time scale $\left(\mathrm{Kn}^{0}\right)$ and the viscosity time scale $\left(\mathrm{Kn}^{1}\right)$, that is, $\Delta / L \sim \mathrm{Kn}^{\gamma}$ with $0<\gamma<1 / 2$ requires a generalization of the ChapmanEnskog method to noninteger series expansion in Knudsen number. This interesting possibility needs to be studied separately. The application of the method of the invariant manifold [17], which does not require Knudsen number $\mathrm{Kn}$ to be small, on the filtered kinetic equation for $0<\gamma<1 / 2$ is a possible extensions of the present work. The possibility of doing exact 
Chapman-Enskog expansion [18,19], also need to be investigated further. Another possible extensions is the use of "renormalization-group" ideas by applying several filters of increasing filter-widths.

- When the discrete-velocity kinetic theory of section (2) is appropriately discretized in time and space, one arrives at the so-called entropic lattice Boltzmann method [2,3] (ELBM). In the ELBM, the thermodynamic stability (Boltzmann's $H$-theorem) is maintained by the discrete-time $H$-theorem $[20,21,22,23]$ which results in unconditionally stable simulation algorithm for hydrodynamics. It was argued [21] that ELBM is a built-in subgrid model. It would be interesting therefore to establish a closer relation between ELBM and the present theory.

- Filtering the kinetic equations as above can be applied to a wide class of kinetic theories with a well-defined separation of time scales enrich existing resolved macroscopic models with physically sound subgrid contribution (for example, the kinetic equations for the granular flows [24]).

To conclude, the presented coarse-grained equations are the first rigorously derived subgrid model. Effectiveness of the model in practice needs to be investigated further numerically. Work in this direction is currently in progress.

\section{$7 \quad$ Acknowledgments}

I. V. K. and S. A. are indebted to A. N. Gorban for many enlightening discussions, and suggestion to lift macroscopic equations to a kinetic theory before coarse-graining. Useful discussions with K. Boulouchos, H. Chen, C. Frouzakis, S. Orszag, H. C. Öttinger, R. Thaokar, and A. Tomboulides are kindly acknowledged.

\section{References}

[1] S. B. Pope, Turbulent Flows (Cambridge University Press, Cambridge, 2000).

[2] I. V. Karlin, A. Ferrante and H. C. Öttinger, Perfect Entropy Functions of the Lattice Boltzmann Method, Europhys. Lett. 47, 182-188 (1999).

[3] S. Ansumali, I. V. Karlin and H. C. Öttinger, Minimal Entropic Kinetic Models for Hydrodynamics, Europhys. Lett. 63 , 798-804 (2003).

[4] J. Smagorinsky, General Circulation Experiments with the Primitive Equations: I. The Basic Equations, Mon. Weather Rev. 91 , 99-164 (1963).

[5] A. Leonard, Energy Cascade in Large-Eddy Simulations of Turbulent Fluid Flows, Adv. Geophys. 18, 237-248 (1974). 
[6] R. A. Clark, J. H. Ferziger, and W. C. Reynolds, Evaluation of Subgrid-Scale Turbulence Models Using an Accurately Simulated Turbulent Flow, J. Fluid. Mech. 91, 1-16 (1979).

[7] Y. Shimomura, A Family of Dynamic Subgrid-Scale Models Consistent with Asymptotic Material Frame Indifference, J. Phys. Soc. Japan 68, 2483-2486 (1999).

[8] R. H. Kraichnan, Eddy Viscosity in Two and Three dimensions J. Atmos. Sci. 33, 1521-1536 (1976).

[9] H. Kobayashi and Y. Shimomura, Inapplicability of the Dynamic Clark Model to the Large Eddy Simulation of Incompressible Turbulent Channel Flows, Phys. Fluids 15, L29-L32 (2003).

[10] S. Chapman and T. Cowling, Mathematical Theory of Non-Uniform Gases (Cambridge University Press, Cambridge, 1970)

[11] H. Chen, S. Succi and S. Orszag, Analysis of Subgrid Scale Turbulence Using the Boltzmann Bhatnagar-Gross-Krook kinetic equation Phys. Rev E 59, R2527R2530 (1999).

[12] S. Succi, I. V. Karlin, H. Chen and S. Orszag, Resummation Techniques in the Kinetic-Theoretical Approach to Subgrid Turbulence Modeling, Physica A 280, 92-98 (2000).

[13] S. Succi, O. Filippova, H. Chen and S. Orszag, Towards a Renormalized Lattice Boltzmann Equation for Fluid Turbulence, J. Stat. Phys 107, 261-278 (2002).

[14] H. Chen, S. Kandasamy, S. Orszag, R. Shock, S. Succi and V. Yakhot, Extended Boltzmann Kinetic Equation for Turbulent Flows, Science 301, 633-636 (2003).

[15] A. Majda, Compressible Fluid Flow and System of Conservation Laws in Several Space Variables (Springer-Verlog, Berlin, 1984).

[16] R. Benzi, Getting a grip on Turbulence, Science 301, 605-606 (2003).

[17] A. N. Gorban, and I. V. Karlin, Method of Invariant Manifolds and Regularization of Acoustic Spectra, Trans. Theory Stat. Phys. 23, 559$632(1994)$.

[18] I. V. Karlin and A. N. Gorban, Hydrodynamics from Grad's Equations: What can We Learn from Exact Solutions? Ann. Phys. (Leipzig) 11(10-11) 783-833 (2002).

[19] M. Slemrod, Renormalization of the Chapman-Enskog Expansion: Isothermal Fluid Flow and Rosenau Saturation, J. Stat. Phys. 91, 285-305(1998).

[20] I. V. Karlin, A. N. Gorban, S. Succi and V. Boffi, Maximum Entropy Principle for Lattice Kinetic Equations, Phys. Rev. Lett. 81, 6-9 (1998).

[21] S. Ansumali and I. V. Karlin, Single Relaxation Time Model for Entropic Lattice Boltzmann Methods, Phys. Rev. E 65, 056312 (2002). 
[22] S. Succi, I. V. Karlin and H. Chen, Role of the H-Theorem in Lattice Boltzmann Hydrodynamic Simulations, Rev. Mod. Phys. 74, 1203-1220 (2002).

[23] B. M. Boghosian, P. J. Love, P. V. Coveney, I. V. Karlin, S. Succi and J. Yepez, Galilean-Invariant Lattice-Boltzmann Models with $\mathrm{H}$-Theorem, Phys. Rev. E 68, 025103(R) (2003).

[24] V. Kumaran, Hydrodynamic Modes of a Sheared Granular Flow from the Boltzmann and the Navier-Stokes Equations, Phys. Fluids 13, 2258-2268 (2001). 